\title{
Cervical rib in 8-year-old Shih-tzu female: Diagnosis and surgical treatment. Case report
}

\author{
ALEKSANDRA KIMICKA ${ }^{1}$, ANNA ŁOJSZCZYK ${ }^{2}$, \\ MAREK BIENKKO ${ }^{1}$, RADOSŁAW P. RADZKI ${ }^{1}$
}

\author{
1Department of Animal Physiology, Faculty of Veterinary Medicine, University of Life Sciences in Lublin, \\ Akademicka 12, 20-950 Lublin, Poland \\ ${ }^{2}$ Laboratory of Radiology and Ultrasonography, Department and Clinic of Animal Surgery, \\ University of Life Sciences in Lublin, Głęboka 30, 20-612, Lublin, Poland
}

Kimicka A., Łojszczyk A., Bieńko M., Radzki R. P.

Cervical rib in 8-year-old Shih-tzu female: Diagnosis and surgical treatment. Case report

Summary

A cervical rib is a rare condition in dogs and cats, but is widely described in humans. It is a congenital disorder involving the presence of additional ribs located mainly on one side or, less often, on both sides of the seventh cervical vertebra. Cervical ribs are mostly found accidentally during chest $\mathrm{X}$-rays. Occasionally, however, the presence of a cervical rib can be symptomatic: neurological, cardiovascular, or respiratory symptoms are possible. An 8-year-old Shih-tzu female was presented with a progressive monoplegia of the left thoracic limb. Computed tomography revealed cervical ribs on both sides of the seventh cervical vertebra. The patient underwent surgery treatment and fully recovered.

Keywords: Cervical rib, dog, surgery, computed tomography, monoplegia

Congenital anomalies of the spine in animals are often diagnosed incidentally, and the first symptoms may appear as the animal ages (4). Cervical ribs occurs mainly in humans, sometimes affects dogs, and can occasionally be found in cats (5). The term "cervical rib" is used to describe an additional rib arising mostly from the seventh cervical vertebra, rarely from the sixth vertebra, and extremely rarely from the fifth vertebra (6). About $10 \%$ of people with a cervical rib will have no symptoms, and only $8 \%$ to $10 \%$ will need surgical treatment (2). Initially, the cervical rib was regarded as a random finding with no clinical significance. Then, the structure of the thorax was explored in greater detail, and the risks posed by the cervical rib were understood. The main risk associated with the presence of the cervical rib is related to the close proximity of important blood vessels and nerves of the brachial plexus. Currently, the genetic basis underlying the occurrence of the cervical rib is assumed (1). It is known that cervical ribs in humans are caused by thoracic outlet syndrome (TOS) (3). TOS leads to symptoms caused by compression of the neurovascular bundle of the neck cranial to the physiologically first rib (8). The cardiovascular bundle of the neck contains three structures: the brachial plexus, the subclavian artery, and the subclavian vein. For this reason, the following types of TOS can be distinguished: arterial, venous, and neurogenic. Thus patients suffering from TOS have neurological or vascular symptoms (7). An 8-year-old female Shih-tzu was presented with a progressive lameness of the left thoracic limb. Neurological examination revealed monoplegia and neurological deficits in the left forelimb. Computed tomography was performed, and cervical ribs were found on both sides of the seventh cervical vertebra, but the right one was greatly reduced. Computed tomography was performed as the primary diagnostic imaging modality because the first suspected neurolocalization was the brachial plexus. In this article the authors focus on the diagnosis and surgical treatment of the cervical rib.

\section{Case report}

An 8-year-old female Shih-tzu (weighing $7 \mathrm{~kg}$ ) was referred to the Center for Small Animals in Lublin because of progressive walking problems that started three days earlier. The owner reported the dog's inability to jump on the furniture, difficulty in walking on stairs, and stumbling. Moreover, the owners noticed that the dog was weak and unwilling to play. No trauma in the past was found. In the neurological examination, proprioceptive ataxia was present, as well as weaker general proprioception and hopping in the left forelimb. Not only postural reactions, but also 


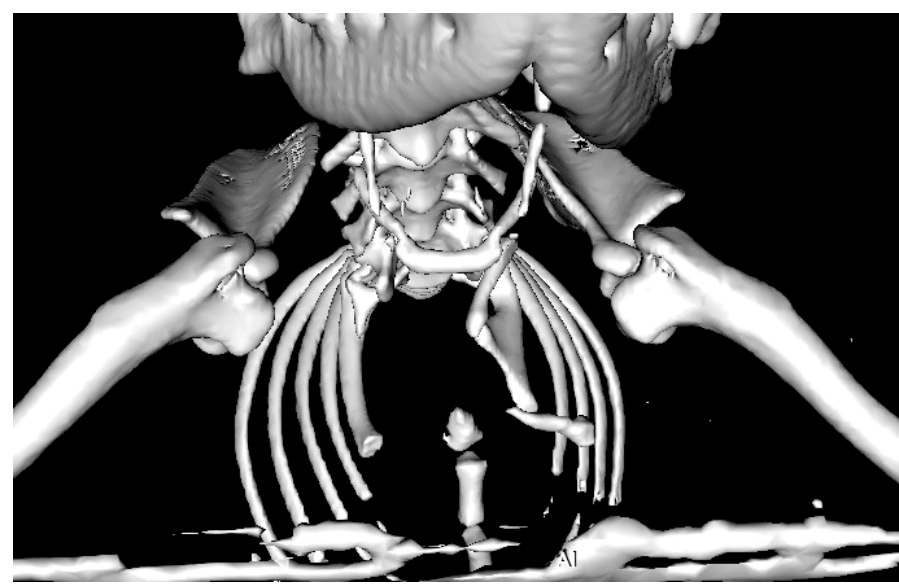

Fig. 1. 3D image reconstruction from a $C T$ scan in the abdominal-dorsal approach

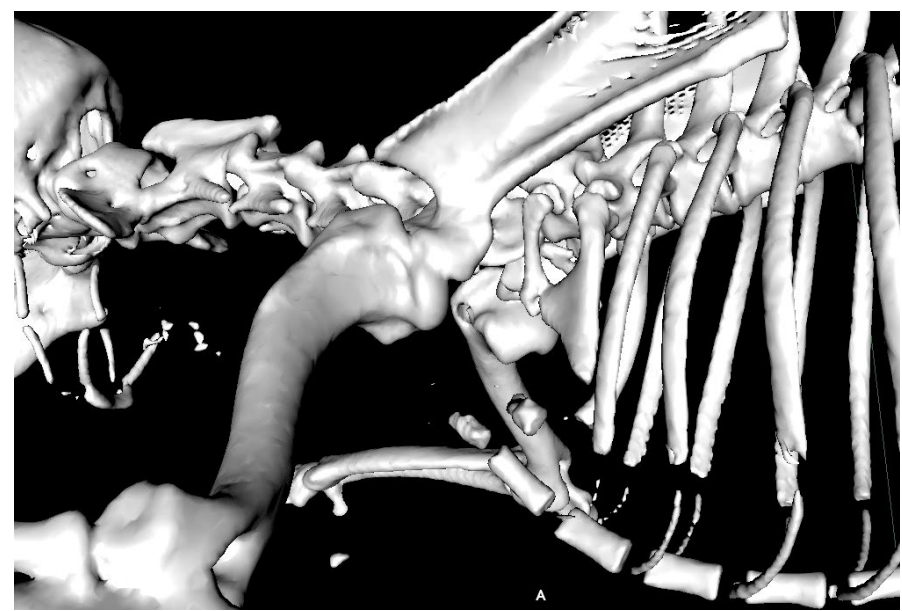

Fig. 2. 3D reconstruction of the image from the CT scan in a lateral view, showing the cervical rib on the left side

spinal reflexes, were disturbed. In the left thoracic limb, the withdrawal reflex was absent and the extensor carpi radialis reflex was weaker. The brachial plexus was suspected as the initial neurolocalization, and the patient referred for computed tomography examination. Differential diagnosis included tumor, an inflammatory process, or, less likely, intervertebral disk disease.

The dog was anaesthetized with Propofol (Scanofol 10 $\mathrm{mg} / \mathrm{ml}$, ScanVet, Poland), and a tomographic examination was performed with a Philips MX-16 slice unit. Images were acquired with the soft algorithm in a soft tissue window. The soft tissue algorithm included unenhanced and contrast-enhanced phases with iv iohexol injection at a dose of $2 \mathrm{ml} / \mathrm{kg}$ BW (Omnipaque $300 \mathrm{mg} / \mathrm{mL}$, GE Healthcare AS, Oslo, Norway). The image acquisition parameters were $120 \mathrm{kV}, 200 \mathrm{mAs} / \mathrm{slice}$, pitch 1.0069, slice thickness 1.0, slice increment $0.5 \mathrm{~mm}$, collimation $16 \times 0.75$, and rotation time $0.75 \mathrm{~s}$. The images were sent to the Philips IntelliSpace Portal and to other computer units, where they were analyzed with the Horos program (GNU Lesser General Public License, Version 3, LGPL-3.0).

CT examination revealed the presence of bilateral cervical ribs of the seventh cervical vertebra. The $7^{\text {th }}$ cervical vertebra has long bilateral transverse processes. The rib on the right side was $11 \mathrm{~mm}$ long. The costovertebral joint was visible, with the costal tubercule connected to the right vertebral transverse process. The head of the rib was slightly displaced ventrally compared to the distal thoracic vertebrae, and it was located in the middle of the vertebral body on its right side at the level of the intervertebral space (C7/Th1) (Fig. 1). The rib on the left side was $17 \mathrm{~mm}$ long. There was no visible head or costal tubercule. The proximal costal part of the left rib was connected to the left transverse process in the costovertebral joint, similar to the right side. The rib in the distal part was fused with the $1^{\text {st }}$ thoracic rib, which was deformed and wide in the ventral part (Fig. 2). At the site of the fusion, the ribs were displaced medially toward the mediastinum. At that location, there was a visible compression and remodeling of the vertebral artery and vein, as well as the deep cervical vein (Fig. 3). The rib was also displaced toward the esophagus, which was laterally flattened and filled with gas at that level (Fig. 4).

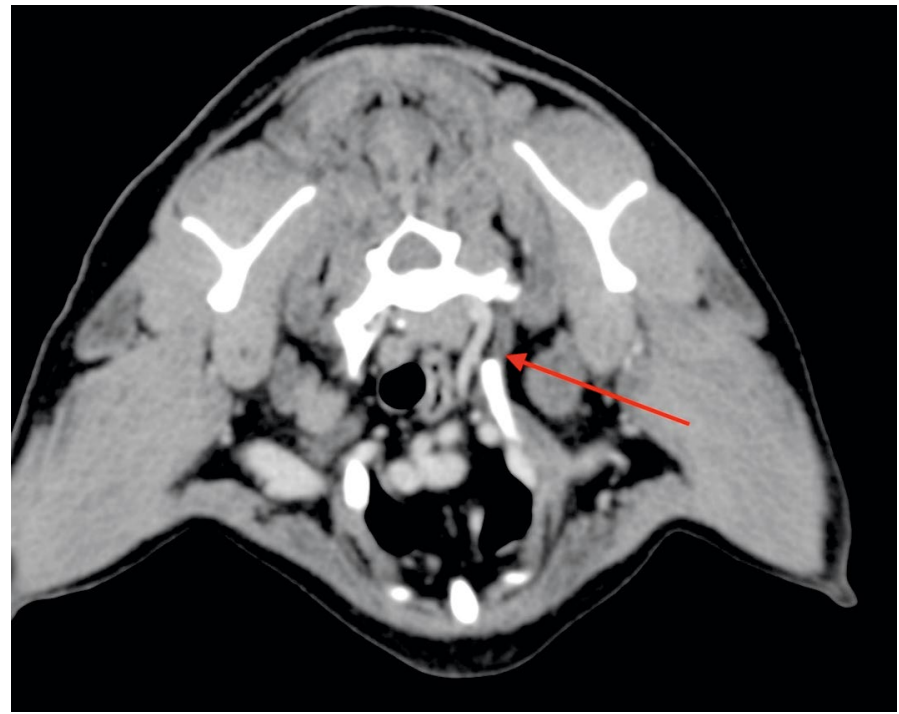

Fig. 3. Soft tissue window, a transverse plane on the level of the $7^{\text {th }}$ cervical vertebrae, examination after contrast medium iv administration. Visible compression of thoracic vessels and the oesophagus by the cervical rib on the left side

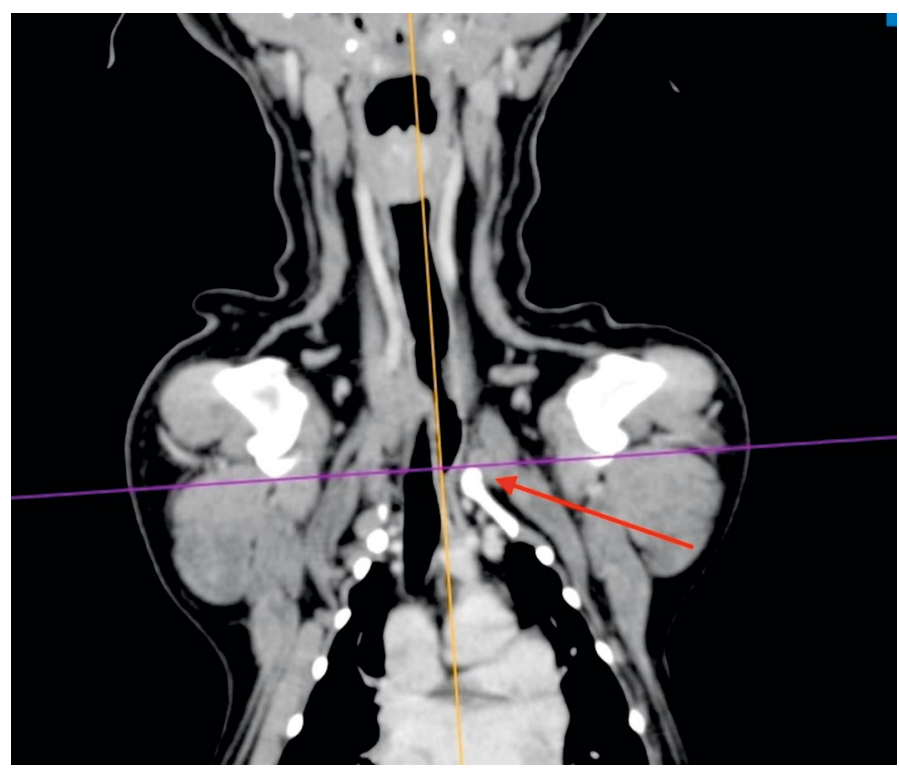

Fig. 4. Soft tissue window, a transverse plane at the level of the $7^{\text {th }}$ cervical vertebrae, examination after contrast medium iv administration. Visible compression of thoracic vessels and the oesophagus by the cervical rib on the left side 
Three weeks later, surgery was performed. The patient was placed under general anesthesia, and the left cervical rib was removed from the midline. Due to complications during anesthesia, the patient was hospitalized. During the first two days after surgery, the patient was lying and unable to walk. General proprioception was delayed in the left thoracic limb, and there was a postoperative oedema of the surgery wound and the left thoracic limb. On the third day after surgery, the dog was able to stand up and her general condition improved. On the following day, the dog started to walk, and there were no neurological deficits in the left thoracic limb. The patient was discharged home with recommendations. No neurological deficits were found during the three-day follow-up visit. The progress of the treatment is presented in Table 1. The patient was referred for physical therapy.

\section{Conclusion}

The cervical rib is usually an incidental finding during imaging diagnostic procedures. However, it can lead to multiple symptoms from the cardiovascular system, respiratory tract, or brachial plexus. A nonsymptomatic cervical rib does not need treatment, but in some cases, it can be the main cause of disease. A cervical rib is often symptomatic at an early age. The patient described in this publication proves that, although the cervical rib is a congenital disorder, signs can develop later in the dog's life. Correct diagnoses
Tab. 1. Comparison of the results of neurological examination before and after cervical rib removal

\begin{tabular}{|l|l|l|}
\hline & \multicolumn{1}{|c|}{ First visit in the clinic } & After cervical rib removal \\
\hline Gait & Proprioceptive ataxia & Normal \\
\hline Hopping & Decreased in the left forelimb & Normal \\
Withdrawal reflex & Incorrect in the left forelimb & Normal \\
\hline Extensor carpi radialis reflex & Decreased in the left forelimb & Normal \\
\hline
\end{tabular}

can lead to treatment that will resolve the patient's problems and dysfunctions.

\section{References}

1. Bokhari R. F., Al-Sayyad M. J., Baeesa S. S.: Prevalence of cervical ribs and elongated transverse processes in Saudi Arabia. Saudi. Med. J. 2012, 33, 66-69.

2. Brewin J., Hill M., Ellis H.: The prevalence of cervical ribs in a London population. Clin. Anat. 2009, 22, 331-336.

3. Cantalamessa A., Martin S., Marchegiani A., Fruganti A., Dini F., Tambella A. M.: Bilateral cervical ribs in a mixed breed dog. J. Vet. Med. Sci. 2017, 79, 1120-1124.

4. Komsta R., Twardowski P., Lisiak B., Dębiak P.: Wady wrodzone kregosłupa u psów, najczęściej spotykane w badaniu radiograficznym. Med. Weter. 2014, 23, 714-719.

5. Newitt A., German A. J., Barr F. J.: Congenital abnormalities of the feline vertebral column. Vet. Radiol. Ultrasound. 2008, 49, 35-41.

6. Ricciardi M., De Simone A., Gernone F., Giannuzzi P.: Bilateral cervical ribs in a Dobermann Pinscher. Vet. Comp. Orthop. Traumatol. 2015, 28, 145-150.

7. Sanders R. J., Hammond S. L.: Management of cervical ribs and anomalous first ribs causing neurogenic thoracic outlet syndrome. J. Vasc. Surg. 2002, 36, 51-56.

8. Sanders R. J., Hammond S. L., Rao N. M.: Thoracic outlet syndrome: a review. Neurologist 2008, 14, 365-373.

Corresponding author: Marek Bieńko PhD, Akademicka 12, 20-950 Lublin, Poland; e-mail: marek.bienko@up.lublin.pl 